\title{
Massive runaway stars in the Small Magellanic Cloud
}

\author{
V. V. Gvaramadze $e^{1,2,3}$, J. Pflamm-Altenburg ${ }^{1}$, and P. Kroupa ${ }^{1}$ \\ 1 Argelander-Institut für Astronomie, Universität Bonn, Auf dem Hügel 71, 53121 Bonn, Germany \\ e-mail: [jpflamm; pavel] @astro.uni-bonn.de \\ 2 Sternberg Astronomical Institute, Moscow State University, Universitetskij Pr. 13, Moscow 119992, Russia \\ e-mail: vgvaram@mx.iki.rssi.ru \\ ${ }^{3}$ Isaac Newton Institute of Chile, Moscow Branch, Universitetskij Pr. 13, Moscow 119992, Russia
}

Received 27 August 2010 / Accepted 10 October 2010

\section{ABSTRACT}

\begin{abstract}
Using archival Spitzer Space Telescope data, we identified for the first time a dozen runaway OB stars in the Small Magellanic Cloud (SMC) through the detection of their bow shocks. The geometry of detected bow shocks allows us to infer the direction of motion of the associated stars and to determine their possible parent clusters and associations. One of the identified runaway stars, AzV 471, was already known as a high-velocity star on the basis of its high peculiar radial velocity, which is offset by $\simeq 40 \mathrm{~km} \mathrm{~s}^{-1}$ from the local systemic velocity. We discuss implications of our findings for the problem of the origin of field OB stars. Several of the bow shockproducing stars are found in the confines of associations, suggesting that these may be "alien" stars contributing to the age spread observed for some young stellar systems. We also report the discovery of a kidney-shaped nebula attached to the early WN-type star SMC-WR3 (AzV 60a). We interpreted this nebula as an interstellar structure created owing to the interaction between the stellar wind and the ambient interstellar medium.
\end{abstract}

Key words. stars: formation - stars: kinematics and dynamics - stars: massive - Magellanic Clouds

\section{Introduction}

Gravitational interaction between massive stars in the cores of young star clusters results in ejection of a significant fraction of these stars from the parent clusters (e.g. Pflamm-Altenburg \& Kroupa 2006; Moeckel \& Bate 2010). The ejected (runaway) stars form the population of field OB stars that end their lives in supernova explosions hundreds of parsecs from their birthplaces. The high velocities of runaway OB stars can be revealed either directly, via measurement of their proper motions and/or radial velocities (e.g. Moffat et al. 1998; Mdzinarishvili \& Chargeishvili 2005; Massey et al. 2005; Evans et al. 2010), or indirectly, through the detection of bow shocks generated ahead of supersonically moving stars (Gvaramadze \& Bomans 2008b; Gvaramadze et al. 2010a, hereafter Paper I). The proper motions can be used to trace the trajectories of the field stars back to their parent clusters, hence to prove their runaway nature (e.g. Hoogerwerf et al. 2001; Schilbach \& Röser 2008). But the proper motions can be measured with a high confidence only for very high-velocity or relatively nearby stars. Measurements of radial velocities can be used to prove the runaway nature of the field stars as well, but taken alone they do not allow us to infer the birth clusters of the stars. For distant OB stars, detection of bow shocks provides a unique possibility not only to show that these stars are runaways, but also to infer the direction of their motion on the sky and thereby to search for their likely parent clusters and OB associations (Gvaramadze \& Bomans 2008b; Paper I).

The necessary conditions for the existence and detection of bow shocks are that the associated stars are moving through the interstellar medium with a supersonic velocity $\left(>10 \mathrm{~km} \mathrm{~s}^{-1}\right.$, in the warm ionized medium) and that the ambient medium is dense enough to ensure a sufficiently high emission measure of the bow shocks (either in $\mathrm{H}_{\alpha}$ or in the infrared). Observations show that $\$ 20$ per cent of runaway OB stars are associated with (detectable) bow shocks (Van Buren et al. 1995; Noriega-Crespo et al. 1997; Huthoff \& Kaper 2002).

Identification of runaway stars via the detection of their bow shocks was used by us to search for OB stars running away from young star clusters in the Milky Way (Gvaramadze \& Bomans 2008a,b; Gvaramadze et al. 2010b) and to prove the runaway nature of the very massive field stars in the Large Magellamic Cloud (LMC, Paper I). In the latter case, we detected bow shocks around several field OB stars (the first-ever detection of bow shocks generated by extragalactic stars), one of which, the $\mathrm{O} 2 \mathrm{~V}\left(\left(\mathrm{f}^{*}\right)\right)$ star BI 237, had been earlier proposed as a candidate runaway star owing to its high peculiar radial velocity (Massey et al. 2005). A logical extension of this work is to use the same approach to identify runaway stars in the Small Magellanic Cloud (SMC), after the LMC our next closest dwarf galaxy, where bow shocks can still be resolved with modern infrared telescopes. In this paper, we present the results of a systematic search for bow shock-producing stars in the SMC, using the Spitzer Space Telescope archival data. Throughout the paper we use a distance of $\simeq 60 \mathrm{kpc}$ for the SMC (Hilditch et al. 2005) so that $1^{\prime}$ corresponds to $\simeq 17 \mathrm{pc}$.

\section{Search for bow shocks in the SMC}

To search for bow shocks in the SMC, we utilized the publicly available imaging data from the Spitzer Space Telescope archive. Using the Leopard software, we retrieved images of the SMC obtained with the Multiband Imaging Photometer for Spitzer (MIPS, Rieke et al. 2004) in the framework of the $S^{3} \mathrm{MC}$ 
Table 1. Summary of bow shock-producing stars in the SMC.

\begin{tabular}{lccccccc}
\hline \hline Star & $\begin{array}{c}\text { RA } \\
(\text { J2000 })\end{array}$ & $\begin{array}{c}\text { Dec } \\
(\text { J2000 })\end{array}$ & $\begin{array}{c}\text { Spectral } \\
\text { type }\end{array}$ & $\begin{array}{c}\text { Ref. } \\
\text { Association } \\
\text { or cluster }\end{array}$ & $\begin{array}{c}\text { Age } \\
(\mathrm{Myr})\end{array}$ & $\begin{array}{c}\text { Separation } \\
(\mathrm{pc})\end{array}$ \\
\hline [MB2000] 37 & 004730.12 & -730507.5 & B9 Ia & 1 & DEM S 47 & $8^{(a)}$ & 210 \\
AzV 26 & 004750.01 & -730821.0 & O6 I(f) & 2 & SMC ASS 13 & $\leq 4^{(a)}$ & 230 \\
[M2002] SMC 9824 & 004802.63 & -731638.7 & OB & 3 & DEM S 45 & $10^{(a)}$ & 160 \\
[M2002] SMC 12977 & 004914.13 & -731442.6 & OB & 3 & DEM S 32 & $10^{(a)}$ & 160 \\
AzV 56 & 004951.26 & -725545.3 & B2.5 Ia & 4 & DEM S 55 & $6^{(a)}$ & 150 \\
AzV 75 & 005032.39 & -725236.5 & O5.5 I(f) & 5 & SMC ASS 22 & $\leq 4^{(a)}$ & 100 \\
AzV 77 & 005033.54 & -724745.0 & O7 III & 6 & DEM S 54 & $8^{(a)}$ & 90 \\
AzV 242 & 010006.88 & -721357.5 & B1 Ia & 4 & DEM S 118 & $8^{(a)}$ & 290 \\
AzV 398 & 010609.81 & -715600.8 & O8.5 If & 7 & NGC 371 & $5^{(a)}$ & 270 \\
AzV 429 & 010759.85 & -720053.9 & O7 V & 6 & [BS95] 134 & - & 150 \\
AzV 471 & 011300.41 & -731704.1 & B0 (III) & 8 & NGC 456 & $10^{(b)}$ & 60 \\
[M2002] SMC 83962 & 013154.97 & -732723.4 & OB & 3 & NGC 602c & $3^{(c)}$ & 70 \\
\hline
\end{tabular}

Notes. ${ }^{(a)}$ Chiosi et al. (2006). ${ }^{(b)}$ Hodge (1983). ${ }^{(c)}$ Massey et al. (2000).

References. (1) Oblak \& Chareton (1981); (2) Massey et al. (2004); (3) Oey, King \& Parker (2004); (4) Lennon (1997); (5) Massey et al. (2009); (6) Garmany et al. (1987); (7) Massey \& Duffy (2001); (8) Evans et al. (2004).

(ID $3316^{1}$, Bolatto et al. 2007) and the SAGE-SMC (ID 40245²) projects. Our search was directed to the detection of arclike structures associated with known OB stars. The typical linear size of bow shocks produced by runaway OB stars is several parsec, which at the distance of the SMC corresponds to the angular size of several tens of arc seconds; we therefore limited ourselves to the search for structures of this angular size. From our experience in the search for bow shocks generated by runaway OB stars (e.g. Paper I), we know that the bow shocks are visible mostly in MIPS $24 \mu \mathrm{m}$ images, so that we utilized the $24 \mu \mathrm{m}$ data alone. The angular resolution of these data of 6 arcsec is comparable to or several times smaller than the expected angular size of bow shocks in the SMC.

Visual inspection of the MIPS $24 \mu \mathrm{m}$ images of the SMC revealed numerous diffuse structures whose arc-like morphology suggests that they could be bow shocks. Using the SIMBAD database $^{3}$ and the VizieR catalogue access tool ${ }^{4}$, we found that most of these structures (see Fig. 1 for four examples) are associated with known OB stars, which strengthens their interpretation as bow shocks. The details of the bow shock-producing stars (listed in order of their RA) are presented in Table 1. The equatorial coordinates of the stars were taken from the 2MASS catalogue (Skrutskie et al. 2006). Column 5 gives the references from which the spectral classification was obtained. Note that the spectral type of [MB2000] 37 was estimated photometrically (Oblak \& Chareton 1981). Similarly, three other stars in the table were identified by Oey et al. (2004) as OB stars on the basis of the UBVR survey data of Massey (2002) for the SMC. Follow-up spectroscopy of these four stars is required to refine their spectral types and thereby to check whether their ages are consistent with the ages of their likely parent clusters and associations (see below).

Figure 2 shows the optical (red band) image of the SMC from the Digitized Sky Survey II (DSS-II, McLean et al. 2000) with the positions of bow shock-producing stars indicated by circles ${ }^{5}$. As expected, the majority of bow shock-producing stars reside in

\footnotetext{
${ }^{1}$ http://celestial.berkeley.edu/spitzer/

2 http://sage.stsci.edu/

${ }^{3}$ http://simbad.u-strasbg.fr/simbad/

${ }^{4}$ http://webviz.u-strasbg.fr/viz-bin/VizieR

5 The image was generated by the NASA's SkyView facility (McGlynn et al. 1996).
}

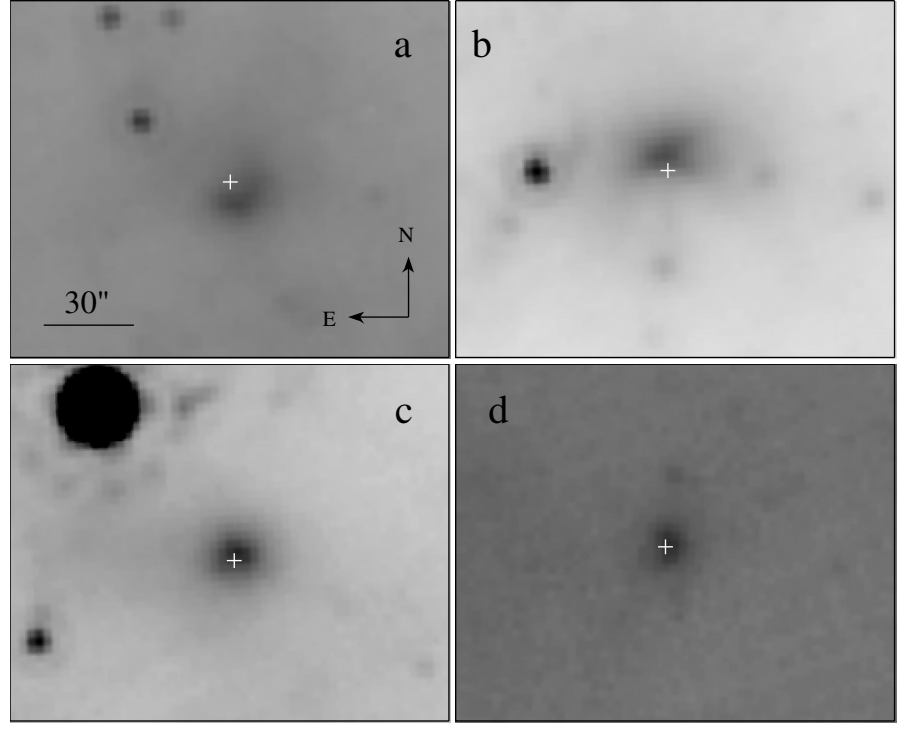

Fig. 1. MIPS $24 \mu \mathrm{m}$ images of four bow shocks associated with a) [MB2000] 37, b) AzV 26, c) AzV 77, and d) AzV 471. The positions of the stars are marked by crosses. The orientation and the scale of the images are the same.

the main body (the bar) of the SMC, where most of star-forming regions and young star clusters are located (e.g., Harris \& Zaritsky 2004). For illustrative purposes, we indicated in Fig. 2 the positions of all 12 known Wolf-Rayet (WR) stars in the SMC, which represent the population of very massive and very young stars (Massey et al. 2000) and therefore closely trace the regions of ongoing star formation. Two of the bow shock-producing stars, namely AzV 471 and [M2002] SMC 83962, were detected in the wing of the SMC (the brightest section of a stellar system extending eastward from the SMC towards the LMC).

To identify possible birthplaces of the bow shock-producing stars, we searched for nearest young ( $\$ 10 \mathrm{Myr})$ star clusters and associations, located in the opposite direction of stellar motion (inferred from the geometry of the bow shocks). The coordinates of clusters and associations and their approximate boundaries were taken from the catalogue by Bica \& Dutra (2000), while the ages were taken from Chiosi et al. (2006), Massey et al. (2000), and Hodge (1983). For one of the associations, 


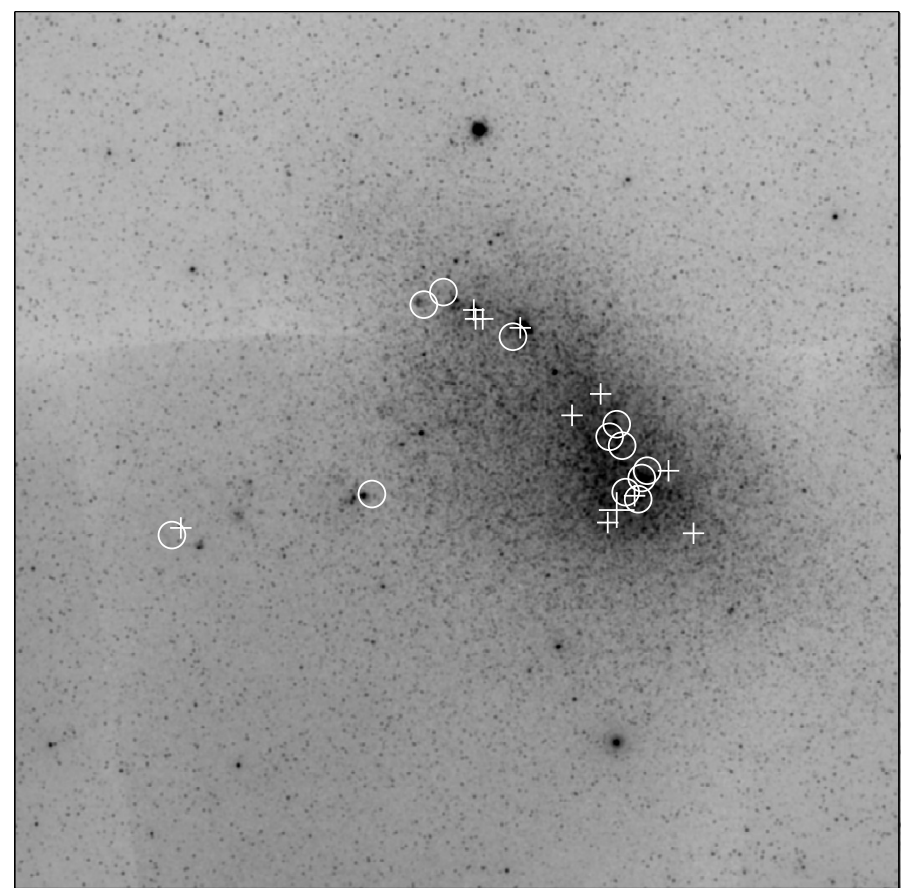

Fig. 2. $6^{\circ} \times 6^{\circ}$ DSS-II image of the SMC and its environments, with the positions of bow shock-producing stars marked by circles. Crosses indicate the positions of all 12 known Wolf-Rayet stars in the SMC, one of which, SMC-WR3, is marked out by a large cross (see text for details). North is up and east to the left.

[BS95] 134, we were unable to find the age estimate. The results from this search are summarized in Table 1, where we give also the separations (in projection) of the stars from the centres of their possible parent clusters and associations, and the ages of the clusters and the associations. Using the figures from the last two columns of Table 1 and assuming that the stars were ejected in the field at the very beginning of dynamical evolution of the parent stellar systems, one can estimate their minimum ejection velocities, i.e., ejection velocity $\left(\mathrm{km} \mathrm{s}^{-1}\right)=$ separation (pc)/age (Myr). For most stars, the (minimum) ejection velocities are quite moderate $\left(<30 \mathrm{~km} \mathrm{~s}^{-1}\right)$, so that they cannot be formally classified as runaways (Blaauw 1961). On the other hand, one cannot exclude the possibility that the detected bow shockproducing stars were ejected from the more distant clusters and associations or that they left their birthplaces after several Myr of cluster evolution (e.g. because of the binary supernova explosions). High-precission proper motion measurements (e.g. with the future space astrometry mission Gaia) are therefore required to resolve the issue.

Figure 3 shows four bow shocks (indicated by solid circles) and the trajectories of their associated stars (shown by arrows), as suggested by the geometry of the bow shocks. The origin of the trajectories corresponds to possible birth associations, whose boundaries are indicated by dashed circles and ellipses. One can see that two stars, AzV 75 and AzV77, are located (at least in projection) within the associations DEM S 54 and DEM S 55, respectively. The detection of the bow shocks generated by these stars (see Fig. 1c for the MIPS $24 \mu \mathrm{m}$ image of the bow shock generated by $\mathrm{AzV} 77$ ) implies that they are runaways and that they therefore were injected into their host associations from the nearby ones, probably from the associations SMC ASS 22 and DEMS 54 (cf. Gvaramadze \& Bomans 2008b; PaperI). The geometry of the bow shock-producing stars, AzV 56 and

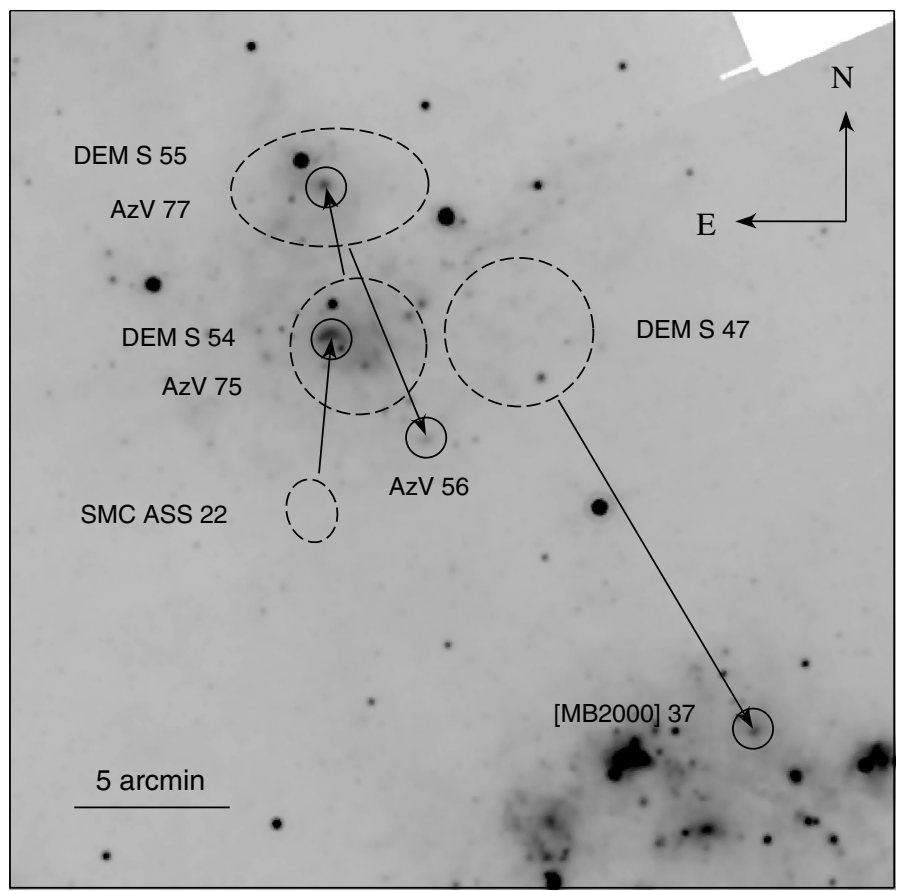

Fig. 3. MIPS $24 \mu \mathrm{m}$ image of the environment of four bow shockproducing stars (indicated by solid circles). The arrows show the direction of motion of the stars, as suggested by the geometry of their bow shocks. The approximate boundaries of the possible birth associations are shown by dashed circles and ellipses (see text for details). At the distance of the SMC, $1^{\prime}$ corresponds to $\simeq 17 \mathrm{pc}$.

[MB2000] 37 (Fig. 1a), suggests that they were ejected from DEM S 55 and DEM S 47, respectively.

Figure 4 shows three other bow shocks and the bow shock (generated by [MB2000] 37) already shown in Fig. 3. The orientation of the bow shocks around [MB2000] 37 (Fig. 1a), AzV 26 (Fig. 1b) and [M2002] SMC 12977 suggests that the first two stars were injected into the association DEM S 32, while the third star, on the contrary, was ejected from the association. The orientation of the bow shock associated with [M2002] SMC 9824 is consistent with the possibility that this star was ejected from DEM S 45.

Figure 5 shows the area to the southeast of the giant starforming region NGC 346. The rich population of very massive stars (including the Luminous Blue Variable/WR binary HD 5980, e.g. Koenigsberger et al. 2010) and significant mass segregation in NGC 346 (e.g., Hennekemper et al. 2008; Sabbi et al. 2008) suggest that this region should be effective in producing runaway stars. It might therefore be expected that some of the ejected stars would manifest themselves in bow shocks. Despite this expectation, we found only one bow shock-producing star around NGC 346, and this star, AzV 242, is instead moving towards NGC 346.

Figures 6-8 show the remaining four bow shock-producing stars detected in the SMC. Two of these stars, AzV 398 and $\mathrm{AzV} 429$, are located near the northeastern edge of the SMC and were probably ejected from the associations, NGC 371 and [BS95] 134, respectively (Fig. 6). The bow shock-producing star AzV 471 is located not far from the N83/N84 starforming region (Henize 1956) of the inner wing of the SMC (Fig. 7). The orientation of the bow shock (see Fig. 1d) is consistent with the possibility that the star was ejected from the association NGC 456. The last bow shock-producing star, [M2002] SMC 83962, is located to the east from the association 


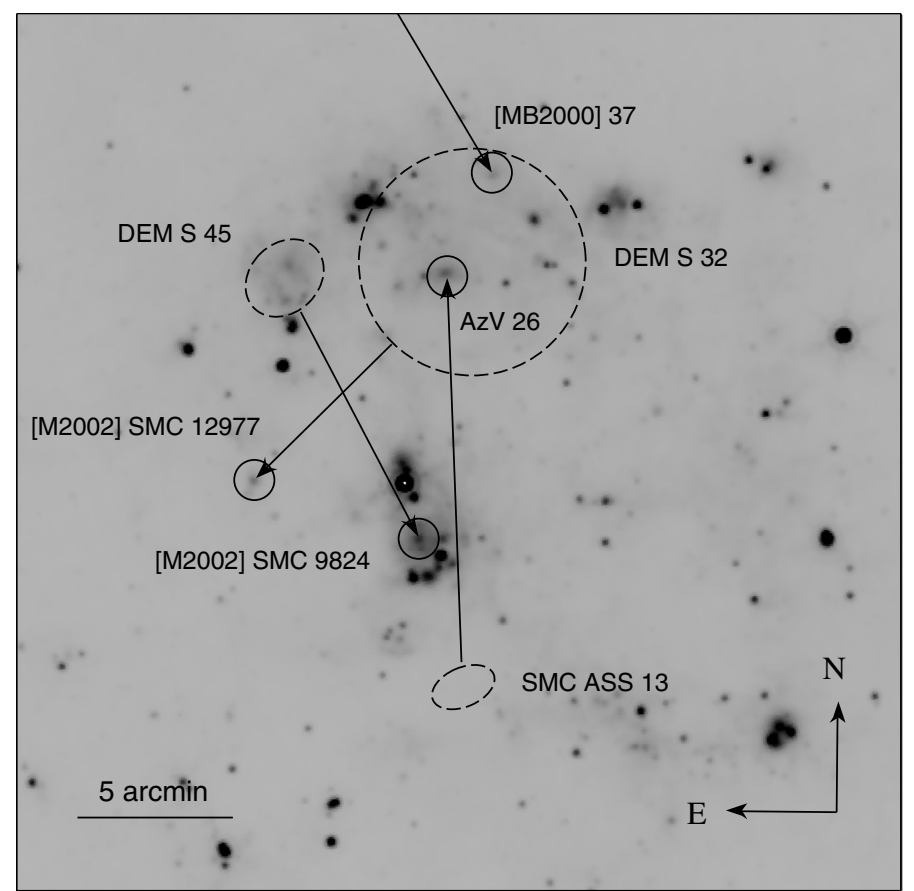

Fig. 4. MIPS $24 \mu \mathrm{m}$ image of the environment of four bow shockproducing stars (indicated by solid circles). The arrows show the direction of motion of the stars, as suggested by the geometry of their bow shocks. The approximate boundaries of the possible birth associations are shown by dashed circles and ellipses.

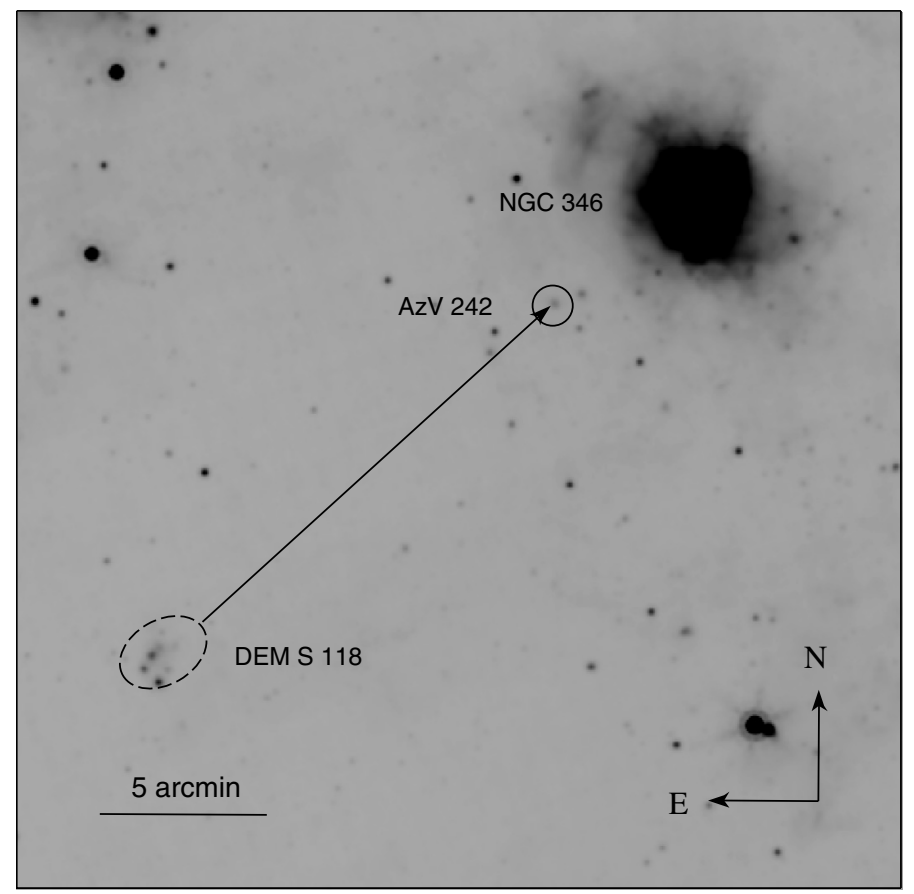

Fig. 5. MIPS $24 \mu \mathrm{m}$ image of the area to the southeast of the giant star-forming region NGC 346 with the bow shock-producing star $\mathrm{AzV} 242$ (indicated by a solid circle) and its possible parent association DEM S 118 (shown by a dashed ellipse). The arrow shows the direction of motion of the star, as suggested by the geometry of the bow shock.

NGC 602c (Fig. 8), which contains the only WO star, SK 188, known in the $\mathrm{SMC}^{6}$. The relative position of the bow shock, the

\footnotetext{
${ }^{6}$ Actualy, SK 188 is a binary system composed of WO4 and O V stars (Foellmi et al. 2003a).
}

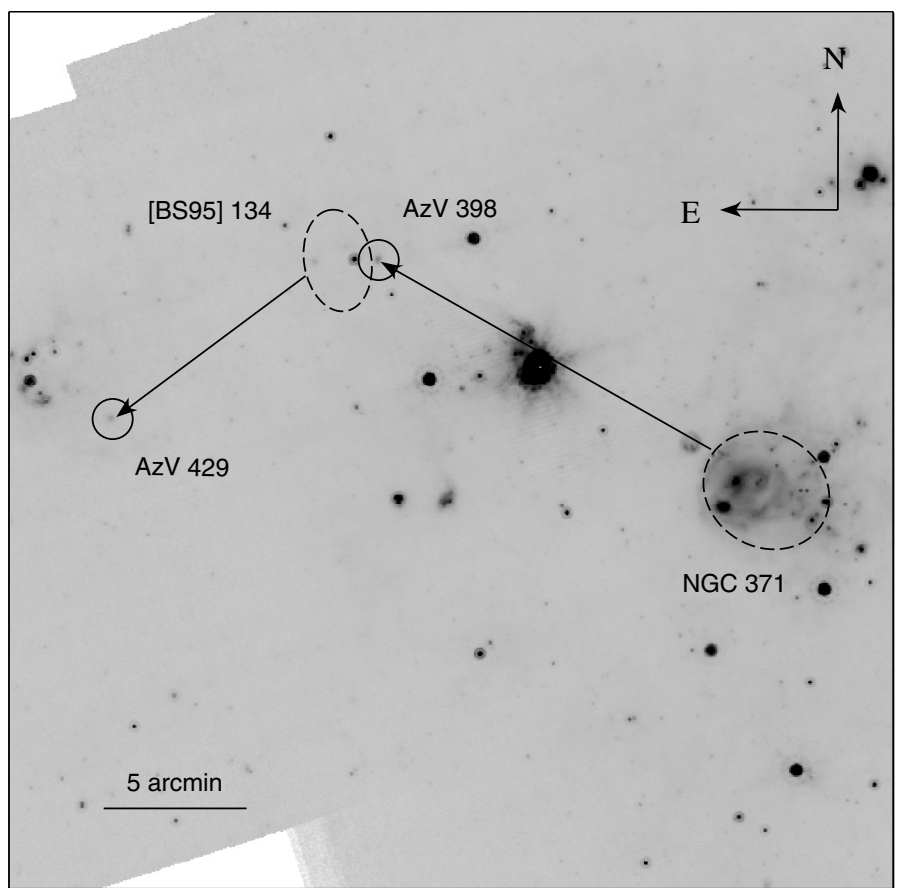

Fig. 6. MIPS $24 \mu \mathrm{m}$ image of the environment of AzV 398 and AzV 429 and their bow shocks (indicated by solid circles). The arrows show the direction of motion of the stars, as suggested by the geometry of their bow shocks. The approximate boundaries of the possible birth cluster NGC 371 and the association [BS95] 134 are shown by dashed ellipses. A small ring-like structure near the northeast edge of NGC 371 is the supernova remnant 1E 0102.2-7219.

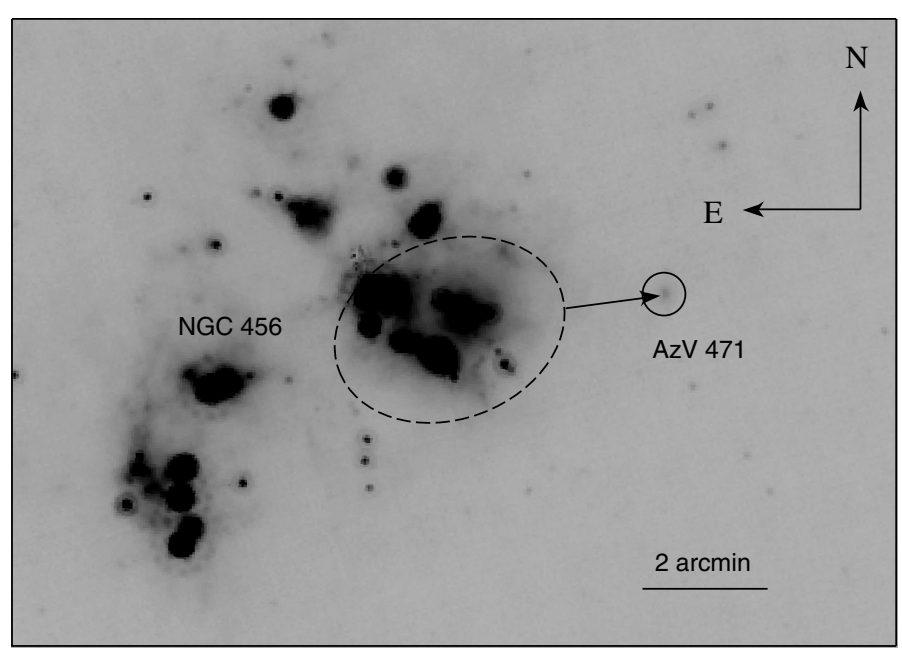

Fig. 7. MIPS $24 \mu \mathrm{m}$ image of the star-forming region N83/N84 with the association NGC 456 (indicated by a dashed ellipse) and the bow shock generated by the BO (III) star AzV 471 (shown by a circle).

star, and the association on the sky are consistent with the possibility that [M2002] SMC 83962 was ejected from NGC 602c.

We also searched for infrared nebulae around all 12 known WR stars in the SMC; see Massey et al. (2003) for a list of these stars, and Fig. 2 for their distribution on the sky. We detected a kidney-shaped nebula attached to the $\mathrm{WN} 3 \mathrm{~h}+\mathrm{O} 9$ (Foellmi et al. 2003a) star SMC-WR3 (also known as AzV 60a; indicated in Fig. 2 by a large cross). The geometry of the nebula (Fig. 9) suggests that it could be either a bow shock or a circumstellar nebula, similar to those produced by the WN6 (Hamann et al. 2006) star WR 136 in the Milky Way and the WN8h 


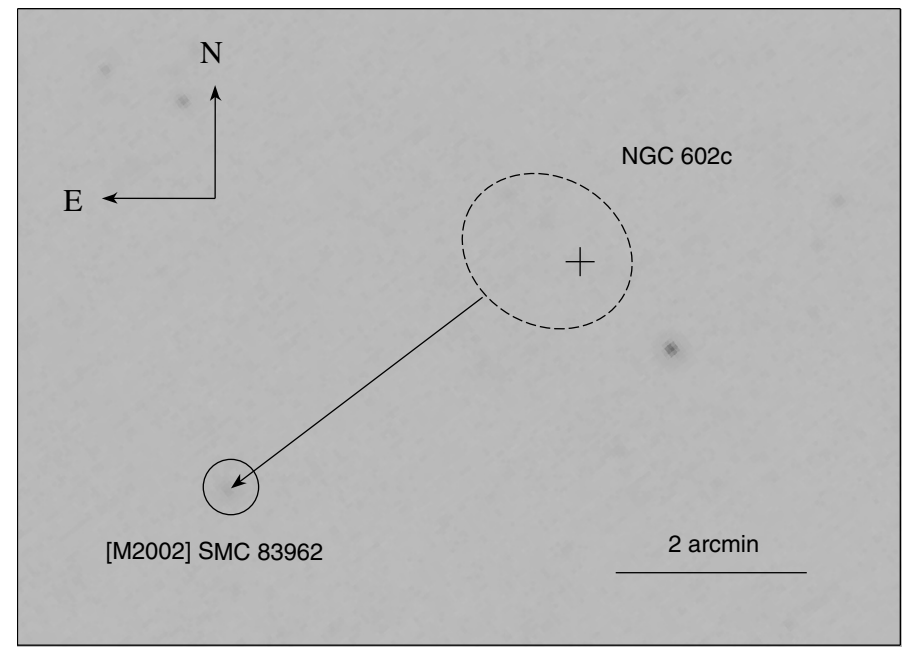

Fig. 8. MIPS $24 \mu \mathrm{m}$ image of the association NGC 602c (indicated by a dashed ellipse) and the bow shock generated by the OB star [M2002] SMC 83962 (shown by a circle). The cross indicates the position of the WO4+O V star SK 188.

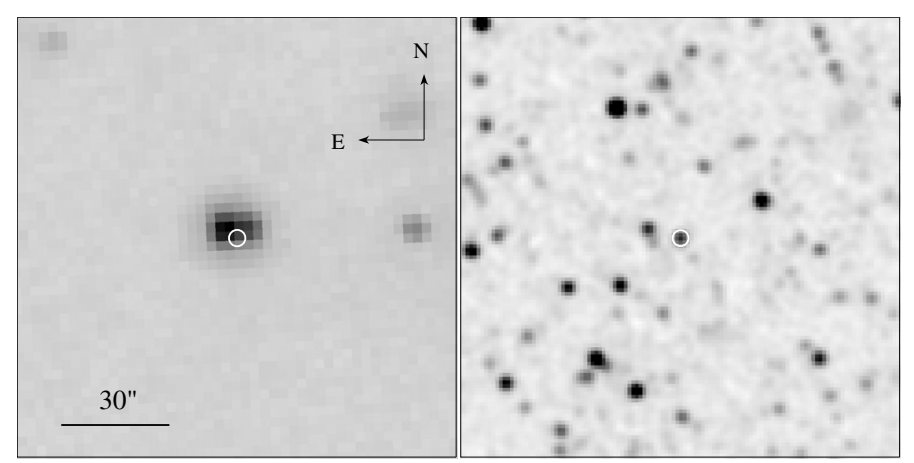

Fig. 9. Left: MIPS $24 \mu \mathrm{m}$ image of a nebula attached to the $\mathrm{WN} 3 \mathrm{~h}+\mathrm{O} 9$ star SMC-WR3 (AzV 60a). The position of SMC-WR3 is marked by a circle. Right: 2MASS J band image of the same field.

(Smith et al. 1996) star Brey 13 in the LMC (see, respectively, Figs. $2 \mathrm{i}$ and q in Gvaramadze et al. 2010c, for the MIPS $24 \mu \mathrm{m}$ images of these nebulae). But because circumstellar nebulae produced by WR stars are observed exclusively around late-type WN stars (Gvaramadze et al. 2010c, and references therein), that is, young WR stars whose wind still interacts with the dense circumstellar material (Gvaramadze et al. 2009a), we inclined to interpret the detected nebula as an interstellar structure, either a bow shock created ahead of the supersonically moving star or a bright rim of a wind-driven bubble blown-up in the inhomogeneous (dense) ambient interstellar medium. Nebulae of similar (linear) size and morphology were detected around the WN2b(h) (Foellmi et al. 2003b) star Brey 2 in the LMC (Chu et al. 1999; see also Fig. 10a for the MIPS $24 \mu \mathrm{m}$ image of the nebula) and the Galactic WN4 (Hamann et al. 2006) star WR 18 (see Fig. 10b for the Midcourse Space Experiment (MSX) satellite, Price et al. 2001, image of the nebula). Both nebulae have normal chemical composition (Garnett \& Chu 1994; Esteban et al. 1993) and therefore are composed of a material swept-up by the stellar wind from the local interstellar medium. We predict that the nebula associated with SMC-WR3 should also have the same abundances as the ambient interstellar medium. Moreover, if the detected nebula is a bow shock, then the possible birthplace of SMC-WR3 is the $\simeq 4$ Myr old association SMC ASS 16 located at 6.7 (or $\simeq 120 \mathrm{pc}$ in projection) to the southwest of the star.

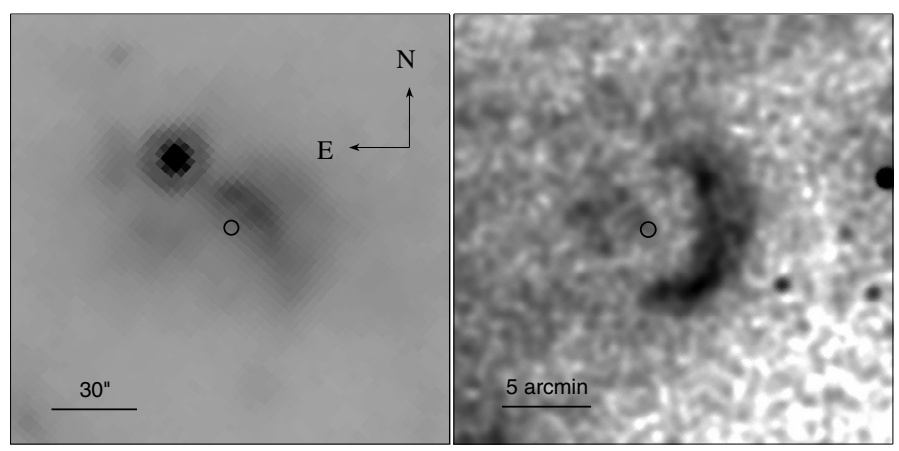

Fig. 10. Left: MIPS $24 \mu \mathrm{m}$ image of a nebula around the WN2b(h) star Brey 2 (indicated by a circle). Right: MSX $14.7 \mu \mathrm{m}$ image of a nebula associated with the WN4 star WR 18 (indicated by a circle).

\section{Discussion and conclusion}

The detection of bow shocks associated with a dozen field OB stars in the SMC unambiguously proves their runaway nature. It is therefore likely that most (and probably all) massive stars in the field are runaways as well (cf. de Wit et al. 2005; Schilbach \& Röser 2008; Gvaramadze \& Bomans 2008b; Pflamm-Altenburg \& Kroupa 2010). Some support for this possibility comes from the observational fact that the velocity dispersion for the O-type stars in the field of the SMC is greater by $\simeq 25$ per cent than that for the $\mathrm{O}$ stars located within $30 \mathrm{pc}$ from the known clusters and associations (Evans \& Howarth 2008).

Another interesting finding by Evans \& Howarth (2008) is that the velocity dispersion for all $\mathrm{O}$ stars in the SMC is larger than that for the less massive (BA) stars. Evans \& Howarth (2008) suggested that this difference is owing to the effect of undetected massive binaries and runaways. This suggestion is consistent with the observational fact that the percentage of runaway stars is highest among the O stars (e.g. Gies 1987; Blaauw 1993; Zinnecker \& Yorke 2007) and that the binary frequency increases with the stellar mass (Larson 2001; Clark et al. 2008).

Like in the Milky Way and the LMC, the runaway nature of some field stars in the SMC can be confirmed via detection of their high peculiar radial velocities. Measurements of radial velocities for $\gtrsim 2000$ stars in the SMC by Evans \& Howarth (2008) show that a significant number of OB stars have heliocentric radial velocities much higher than the SMC's systemic velocity, so that one can expect that at least some of these stars are runaways (cf. Lamb \& Oey 2008). The sample of stars studied by Evans \& Howarth (2008) contains only one of the dozen of bow shock-producing stars listed in our Table 1. Interestingly, the radial velocity of this star, $\mathrm{AzV} 471$, is offset by $\simeq 40 \mathrm{~km} \mathrm{~s}^{-1}$ from the local systemic velocity of the star-forming region N83/N84, where the star is situated. This offset along with the detection of the bow shock generated by $\mathrm{AzV} 471$ undoubtedly proves the runaway nature of the star. Thus, one might expect that measurements of radial velocities for other stars from our list would result in the detection of similar offsets as well.

In our search for bow shock-producing stars we found that some of them are located (at least in projection) within the confines of known associations. The presence of bow shocks around these stars, however, implies that they are runaways and therefore were instead injected into their host associations from the nearby ones (cf. Gvaramadze \& Bomans 2008b; Paper I). From this it follows that the contamination of the stellar content of associations by "alien" OB stars may contribute to the age spread observed for some young stellar systems (e.g. Massey 2003). 
To conclude, we note that searches for bow shocks with the next generation of space infrared telescopes along with future high-precision proper motion measurements for massive field stars with the space astrometry mission Gaia will allow us to solve the problem of whether the massive stars form solely in the clustered mode and subsequently leave their parent clusters because of the gravitational interaction with other massive stars (Poveda et al. 1967; Leonard \& Duncan 1990; see also Kroupa 1998; Pflamm-Altenburg \& Kroupa 2006; Gvaramadze et al. 2009b; Gvaramadze \& Gualandris 2010), or whether they can also form in situ (in the field and/or in low-mass clusters; e.g. Oey et al. 2004; Parker \& Goodwin 2007).

Acknowledgements. We are grateful to the referee for a comment allowing us to improve the presentation of the paper. V.V.G. acknowledges financial support from the Deutsche Forschungsgemeinschaft. This research has made use of the NASA/IPAC Infrared Science Archive, which is operated by the Jet Propulsion Laboratory, California Institute of Technology, under contract with the National Aeronautics and Space Administration, the NASA's SkyView facility (http:// skyview.gsfc.nasa.gov) located at NASA Goddard Space Flight Center, the SIMBAD database, and the VizieR catalogue access tool, both operated at CDS, Strasbourg, France.

\section{References}

Bica, E., \& Dutra, C. M. 2000, AJ, 119, 1214

Blaauw, A. 1961, Bull. Astron. Inst. Netherlands, 15, 265

Blaauw, A. 1993, in Massive Stars: Their Lives in the Interstellar Medium, ed. J. P. Cassinelli, \& E. B. Churchwell, ASP Conf. Ser., 35, 207

Bolatto, A. D., Simon, J. D., Stanimirovic, S., et al. 2007, ApJ, 655, 212

Chiosi, E., Vallenari, A., Held, E. V., Rizzi, L., \& Moretti, A. 2006, A\&A, 452, 179

Chu, Y.-H., Weis, K., \& Garnett, D. R. 1999, AJ, 117, 1433

Clark, J. S., Muno, M. P., Negueruela, I., et al. 2008, A\&A, 477, 147

de Wit, W. J., Testi, L., Palla, F. \& Zinnecker, H. 2005, A\&A, 437, 247

Esteban, C., Vilchez, J. M., Smith, L. J., \& Clegg, R. E. S. 1993, A\&A, 272, 299

Evans, C. J., \& Howarth, I. D. 2008, MNRAS, 386, 826

Evans, C. J., Howarth, I. D., Irwin, M. J., Burnley, A. W., \& Harries, T. J. 2004, MNRAS, 353, 601

Evans, C. J., Walborn, N. R., Crowther, P. A., et al. 2010, ApJ, 715, L74

Foellmi, C., Moffat, A. F. J., \& Guerrero, M. A. 2003a, MNRAS, 338, 360

Foellmi, C., Moffat, A. F. J., \& Guerrero, M. A. 2003b, MNRAS, 338, 1025

Garmany, C. D., Conti, P. S., \& Massey, P. 1987, AJ, 93, 1070

Garnett, D. R., \& Chu, Y.-H. 1994, PASP, 106, 626

Gies, D. R. 1987, ApJS, 64, 545

Gvaramadze, V. V., \& Bomans, D. J. 2008a, A\&A, 485, L29

Gvaramadze, V. V., \& Bomans, D. J. 2008b, A\&A, 490, 1071

Gvaramadze, V. V., \& Gualandris, A. 2010, MNRAS, in press [arXiv: 1007.5057$]$

Gvaramadze, V. V., Fabrika, S., Hamann, W.-R., et al. 2009a, MNRAS, 400, 524

Gvaramadze, V. V., Gualandris, A., \& Portegies Zwart, S. 2009b, MNRAS, 396,570
Gvaramadze, V. V., Kroupa, P., \& Pflamm-Altenburg, J. 2010a, A\&A, 519, A33 (Paper I)

Gvaramadze, V. V., Kniazev, A. Y., Hamann, W.-R., et al. 2010b, MNRAS, 403, 760

Gvaramadze, V. V., Kniazev, A. Y., \& Fabrika, S. 2010c, MNRAS, 405, 1047

Hamann, W.-R., Gräfener, G., \& Liermann, A. 2006, A\&A, 457, 1015

Harris, J., \& Zaritsky, D. 2004, AJ, 127, 1531

Henize, K. G. 1956, ApJS, 2, 315

Hennekemper, E., Gouliermis, D. A., Henning, T., Brandner, W., \& Dolphin, A. E. 2008, ApJ, 672, 914

Hilditch, R. W., Howarth, I. D., \& Harries, T. J. 2005, MNRAS, 357, 304

Hodge, P. W. 1983, ApJ, 264, 470

Hoogerwerf, R., de Bruijne, J. H. J., \& de Zeeuw, P. T. 2001, A\&A, 365, 49

Huthoff, F., \& Kaper, L. 2002, A\&A, 383, 999

Koenigsberger, G., Georgiev, L., Hillier, D. J., et al. 2010, AJ, 139, 2600

Kroupa, P. 1998, MNRAS, 298, 231

Lamb, J. B., \& Oey, M. S. 2008, in Dynamical Evolution of Dense Stellar Systems, ed. E. Vesperini, M. Giersz, \& A. Sills, Proc. IAU Symp., 246, 63

Larson, R. B. 2001, in The Formation of Binary Stars, ed. H. Zinnecker, \& R. D.

Mathieu, Proc. IAU Symp., 200, 93

Lennon, D. J. 1997, A\&A, 317, 871

Leonard, P. J. T., \& Duncan, M. J. 1990, AJ, 99, 608

Massey, P. 2002, ApJS, 141, 81

Massey, P. 2003, ARA\&A, 41, 15

Massey, P., \& Duffy, A. S. 2001, ApJ, 550, 713

Massey, P., Waterhouse, E., \& DeGioia-Eastwood, K. 2000, AJ, 119, 2214

Massey, P., Olsen, K. A. G., \& Parker, J. W. 2003, PASP, 115, 1265

Massey, P., Bresolin, F., Kudritzki, R.-P., Puls, J., \& Pauldrach, A. W. A. 2004, ApJ, 608, 1001

Massey, P., Puls, J., Pauldrach, A. W. A., et al. 2005, ApJ, 627, 477

Massey, P., Zangari, A. M., Morrell, N. I., et al. 2009, ApJ, 692, 618

McGlynn, T., Scollick, K., \& White, N. 1996, in New Horizons from MultiWavelength Sky Surveys, ed. B. J. McLean et al., Proc. IAU Symp., 179, 465

McLean, B. J., Greene, G. R., Lattanzi, M. G., \& Pirenne B. 2000, in Astronomical Data Analysis Software and Systems IX, ed. N. Manset, C. Veillet, \& D. Crabtree, ASP Conf. Ser., 216, 145

Mdzinarishvili, T. G., \& Chargeishvili, K. B. 2005, A\&A, 431, L1

Moeckel, N., \& Bate, M. R. 2010, MNRAS, 404, 721

Moffat, A. F. J., Marchenko, S. V., Seggewiss, W., et al. 1998, A\&A, 331, 949

Noriega-Crespo, A., Van Buren, D., \& Dgani, R. 1997, AJ, 113, 780

Oblak, E., \& Chareton, M. 1981, A\&AS, 45, 459

Oey, M. S., King, N. L., \& Parker, J. W. 2004, AJ, 127, 1632

Parker, R. J., \& Goodwin, S. P. 2007, MNRAS, 380, 1271

Pflamm-Altenburg, J., \& Kroupa, P. 2006, MNRAS, 373, 295

Pflamm-Altenburg, J., \& Kroupa, P. 2010, MNRAS, 404, 1564

Poveda, A., Ruiz, J., \& Allen, C. 1967, Bol. Obs. Tonantzintla Tacubaya, 4, 86

Price, S. D., Egan, M. P., Carey, S. J., Mizuno, D. R., \& Kuchar, T. A. 2001, AJ, 121,2819

Rieke, G. H., Young, E. T., Engelbracht, C. W., et al. 2004, ApJS, 154, 25

Sabbi, E., Sirianni, M., Nota, A., et al. 2008, AJ, 135, 173

Schilbach, E., \& Röser, S. 2008, A\&A, 489, 105

Skrutskie, M. F., Cutri, R. M., Stiening, R., et al. 2006, AJ, 131, 1163

Smith, L. F., Shara, M. M., \& Moffat, A. F. J. 1996, MNRAS, 281, 163

Van Buren, D., Noriega-Crespo, A., \& Dgani, R. 1995, AJ, 110, 2914

Zinnecker, H., \& Yorke, H. W. 2007, ARA\&A, 45, 481 